\title{
Canonical Formulation of IIB D-branes
}

\author{
KiYoshi Kamimura And Machiko HatsudA ${ }^{\dagger}$ \\ Department of Physics, Toho University \\ Funabashi, 274-8501 JAPAN \\ $\dagger$ Department of Radiological Sciences, \\ Ibaraki Prefectural University of Health Sciences \\ Ami, Inashiki, Ibaraki, 300-0394 JAPAN \\ kamimura@ph.sci.toho-u.ac.jp, hatuda@ipu.ac.jp
}

\begin{abstract}
We find Wess-Zumino actions for kappa invariant type IIB D-branes in explicit forms. A simple and compact expression is obtained by the use of spinor variables which are defined as power series of differential forms. Using the Wess-Zumino actions we develop the canonical formulation and find the complete set of the constraint equations for generic type IIB D-p-branes. The conserved global supersymmetry charges is determined and the algebra containing the central charges can be obtained explicitly.
\end{abstract}

hep-th.9712068

December 1997

Revised in April 1998

TOHO-FP-9757 


\section{Introduction}

The superstring theories凹 are promising approaches to the unified theory. In their non-perturvative approaches D-branes play important roles [2] [3]. D-branes, as well as superstrings, are characterized by the diffeomorphism, the local supersymmetries (kappa symmetry) and the global super-Poincaré invariance. Kappa invariant D-brane actions have been proposed in [4] [5] [7] [8]. The action of the D-branes consists of two parts, the Dirac-Born-Infeld (DBI) action and the Wess-Zumino (WZ) action. The DBI lagrangian is manifestly symmetric under SUSY transformation while the WZ lagrangian is pseudo symmetric, i.e. invariant up to total derivative terms. Under the kappa transformation only the sum of DBI and WZ actions remain invariant.

In this paper we develop the canonical formulation of the D-p-branes for generic $p$. It is the first step towards the quantum theories; in canonical, path integral, BRST quantizations and so on. It has been discussed the canonical quantization in connection with the covariant gauge fixing for D-particle and D-string [9] 10].

The defining equation of the WZ action for type IIB D-p-brane in the flat background is, using the notation of [5], as

$$
d L^{W Z}=\mathrm{T} d \bar{\theta} \mathcal{S}_{B} \tau_{1} d \theta e^{\mathcal{F}} .
$$

The integrability is guaranteed by the Bianchi identity for the Ramond/Ramond curvature $R=d \bar{\theta} \mathcal{S}_{B} \tau_{1} d \theta$ and the WZ action is determined up to exact forms. The WZ action $L_{p+1}^{W Z}$ of the IIB D-p-brane is the $(p+1)$ form part of the WZ action $L^{W Z}$. (1.1) would be sufficient in most purposes; the local and global supersymmetry invariances can be proven using only the defining equation. In the case of super p-brane the algebras of covariant derivatives and SUSY were determined from the Ramond/Ramond curvature by using the covariance under supertranslations [11]. However the integrated form for $L^{W Z}$ is often desirable. In the canonical quantization the form of the WZ action is required. For the general D-p-branes it had not been fully studied since the explicit form of the WZ action was not available [9] 12]. In order to perform the canonical analysis for the general D-p-branes, we obtain the WZ action in explicit form in this paper.

Integration of the differential equation (1.1) is not straightforward except for small p. Finding out appropriate variables are essential to perform this integration. In this paper we introduce new spinor variables, $\Theta_{C}$ and $\Theta_{S}$, which are power series of the differential forms. After integrating (1.1), the resultant WZ action is obtained as

$$
L^{W Z}=\mathrm{T}\left(\bar{\Theta}_{C} \mathcal{S}_{B}+\bar{\Theta}_{S} \mathcal{C}_{B}\right) \tau_{1} e^{\mathcal{F}} d \theta
$$

Using this we introduce canonical momenta and derive the complete set of constraint equations for the IIB D-p-branes. We also derive the conserved SUSY charges and compute the SUSY algebra and the central charges for the IIB D-p-branes as functions of world volume variables. As was discussed in [13] [11] the SUSY algebras of extended objects can induce central extensions due to the pseudo-invariance of the WZ action under the SUSY transformations. The SUSY algebras exhibit their BPS properties and discussions of the central charges are one of the important current interests [14, 15, 16]. Local and global

\footnotetext{
${ }^{1}$ See [1] for the review.
} 
supersymmetries of super D-1-brane are deeply related as demonstrated in the reference [10]. Such relation can be examined for D-p-branes with arbitrary p, once explicit forms of kappa constraints, SUSY charges and their algebras are determined. These analysis will give the correct treatments of fermionic degrees of freedom leading to quantum D-brane theories.

In section 2 we introduce some crucial definitions and formulas and integrate (1.1) to find the WZ action $L^{W Z}$ (1.2). In section 3 the constraint equations in the Hamiltonian formalism are derived. The algebra of fermionic constraints is computed and the kappa symmetry of the system is assured in section 4 . In section 5 we show the quasi invariance of the WZ lagrangian under the SUSY transformation. The super symmetry charges are constructed by utilizing the the surface term of the SUSY transformation. In section 6 the global supersymmetry algebra is computed and the expressions of the central charges are presented. In the last section we give short summary and discussions. We add some detailed formulas in Appendix for convention.

\section{WZ action}

The actions of the super D-branes are described by the 10D target space vector coordinates $X^{m},(m=0, \ldots, 9)$, Majorana-Weyl spinors $\theta_{A}^{\alpha},(A=1,2)$ and $\mathrm{U}(1)$ gauge field $A_{\mu},(\mu=0, . ., p)$. In this paper we consider general type IIB theory ( $\mathrm{p}=$ odd $)$ and the two Majorana-Weyl spinors $(A=1,2)$ have the same chirality. The action is constructed from global SUSY invariant forms. They are $d \theta$,

$$
\Pi^{\ell} \equiv d X^{\ell}+\bar{\theta} \Gamma^{\ell} d \theta \equiv d \sigma^{\mu} \Pi_{\mu}^{\ell}
$$

and

$$
\mathcal{F} \equiv d A+\bar{\theta} \tau_{3} \Gamma_{\ell} d \theta\left(\Pi^{\ell}-\frac{1}{2} \bar{\theta} \Gamma^{\ell} d \theta\right) \equiv \frac{1}{2} d \sigma^{\mu} d \sigma^{\nu} \mathcal{F}_{\mu \nu}
$$

We follow the notations of [5] except their $\psi$ is denoted as $\Pi=\Pi^{m} \Gamma_{m}$. Remember the spinors anticommute with odd forms.

The DBI lagrangian density

$$
\mathcal{L}^{D B I}=-\mathrm{T} \sqrt{-\operatorname{det}\left(G_{\mu \nu}+\mathcal{F}_{\mu \nu}\right)} .
$$

is invariant under global SUSY since the induced metric $G_{\mu \nu}=\Pi_{\mu}^{m} \Pi_{m \nu}$ and U(1) field strength $\mathcal{F}$ are invariant. On the other hand the SUSY transformation of the WZ lagrangian density is a total divergence. It is seen from (1.1) that $d L^{W Z}$ is given by a SUSY invariant form. In this section we solve the (1.1) to find the explicit form of $L^{W Z}$.

As preparations we give some useful definitions and formulas. First one is $\mathcal{S}_{B}$ and $\mathcal{C}_{B}$ introduced in [5]

$$
\begin{aligned}
& \mathcal{S}_{B}(\not \Pi)=\sum_{\ell=0} \tau_{3}^{\ell} \frac{\Pi^{2 \ell+1}}{(2 \ell+1) !}=\Pi 1+\tau_{3} \frac{\Pi^{3}}{3 !}+\frac{\Pi^{5}}{5 !}+\ldots, \\
& \mathcal{C}_{B}(\not \Pi)=\sum_{\ell=0} \tau_{3}^{\ell+1} \frac{\Pi^{2 \ell}}{(2 \ell) !}=\tau_{3}+\frac{\Pi^{2}}{2 !}+\tau_{3} \frac{\Pi^{4}}{4 !}+\ldots .
\end{aligned}
$$


Second is $2 \times 2$-matrix valued 10D vector defined for any type IIB spinors $\psi$ and $\phi$

$$
V_{\psi, \phi}^{m} \equiv\left(\bar{\psi} \Gamma^{m} \phi\right)+\tau_{3}\left(\bar{\psi} \tau_{3} \Gamma^{m} \phi\right) .
$$

Using this notation the cyclic identity holds as

$$
V_{\psi, \phi} \theta+V_{\phi, \theta} \psi+V_{\theta, \psi} \phi=0
$$

for three odd IIB spinors. It frequently appears an expression

$$
V^{m} \equiv V_{\theta, d \theta}^{m}=\left(\bar{\theta} \Gamma^{m} d \theta\right)+\tau_{3}\left(\bar{\theta} \tau_{3} \Gamma^{m} d \theta\right) .
$$

The cyclic identity (2.7) tells, for example,

$$
d V \theta+2 \bigvee d \theta=0, \quad \rightarrow \quad d V d \theta=0, \quad V \cdot d V=0 .
$$

Third is j-form IIB spinor $\Theta_{j}$ defined by

$$
\Theta_{j} \equiv V \Theta_{j-1}=\ldots=V^{j} \Theta_{0}, \quad \Theta_{0} \equiv \theta .
$$

It has the $j+1$ parity . It holds an important formula

$$
d \Theta_{j}=\frac{2 j+1}{2} d V \Theta_{j-1}, \quad(j=1,2, \ldots)
$$

which is proved by induction ( see Appendix (A.25)).

The important quantities describing the D-branes are odd IIB spinor form $\Theta_{S}$ and even one $\Theta_{C}$ defined by

$$
\begin{aligned}
\Theta_{S} & \equiv \sum_{n=0} \frac{1}{(4 n+3) ! !}\left(-\tau_{3}\right)^{n+1} \Theta_{2 n+1}=-\frac{1}{3 ! !} \tau_{3} V \theta+\frac{1}{7 ! !} V^{3} \theta-\ldots \\
\Theta_{C} & \equiv \sum_{n=0} \frac{1}{(4 n+1) ! !}\left(-\tau_{3}\right)^{n} \Theta_{2 n}=\theta-\frac{1}{5 ! !} \tau_{3} V^{2} \theta+\ldots
\end{aligned}
$$

Using (2.11) they are shown to satisfy

$$
d \Theta_{C}=d \theta+\frac{1}{2} d V \Theta_{S}, \quad d \Theta_{S}=-\frac{1}{2} \tau_{3} d V \Theta_{C} .
$$

Here $d \theta$ term appears from $\mathrm{n}=0$ term.

The last is $\mathcal{S}_{1}$ and $\mathcal{C}_{1}$, which are respectively $T_{B}$ and $\rho_{B}$ of ref. [5],

$$
\mathcal{S}_{1}=\mathcal{S}_{B} \tau_{1} e^{\mathcal{F}}, \quad \mathcal{C}_{1}=\mathcal{C}_{B} \tau_{1} e^{\mathcal{F}} .
$$

They satisfy, using (A.17),

$$
\begin{aligned}
& d \mathcal{S}_{1}=\frac{1}{2} \tau_{3}\left[d V \mathcal{C}_{1}+\mathcal{C}_{1} d V\right] \\
& d \mathcal{C}_{1}=\frac{1}{2}\left[d \bigvee \mathcal{S}_{1}-\mathcal{S}_{1} d V\right] .
\end{aligned}
$$


Now we are ready to integrate (1.1)

$$
d L^{W Z}=\quad+\mathrm{T} d \bar{\theta} \mathcal{S}_{1} d \theta
$$

to find the $L^{W Z}$. We first write the right hand side of $(2.16)$ as

$$
d \bar{\theta} \mathcal{S}_{1} d \theta=d L_{1}+I_{1}, \quad L_{1}=\bar{\theta} \mathcal{S}_{1} d \theta, \quad I_{1}=\bar{\theta} d\left(\mathcal{S}_{1} d \theta\right) .
$$

Using (2.15), (2.9) and 2.11)

$$
I_{1}=\bar{\theta} \frac{1}{2} d \bigvee \tau_{3} \mathcal{C}_{1} d \theta=d\left(-\frac{1}{3} \bar{\theta} \bigvee\right) \tau_{3} \mathcal{C}_{1} d \theta \equiv d L_{2}+I_{2}
$$

with

$$
L_{2}=\frac{1}{3} \bar{\Theta}_{1}\left(-\tau_{3}\right) \mathcal{C}_{1} d \theta, \quad I_{2}=\frac{1}{3} \bar{\Theta}_{1} \tau_{3} d\left(\mathcal{C}_{1} d \theta\right)
$$

Repeating this procedure ( actually it terminates at $(p+1)$-th step for the $p$ brane ) and summing up $L_{j}$ 's we arrive at a compact expression for the WZ action. It is, up to a total exterior derivative term,

$$
L^{W Z}=\mathrm{T}\left(\bar{\Theta}_{C} \mathcal{S}_{1}+\bar{\Theta}_{S} \mathcal{C}_{1}\right) d \theta
$$

where $\Theta_{S}$ and $\Theta_{C}$ are ones defined in (2.12). The WZ actions of the D-p-brane is the $(p+1)$ form part of $(2.20)$.

It is straightforward to confirm (2.16) by using (2.13) and (2.15):

$$
\begin{aligned}
d L^{W Z} & =\mathrm{T}\left[\left(d \bar{\theta}-\frac{1}{2} \bar{\Theta}_{S} d V\right) \mathcal{S}_{1}-\bar{\Theta}_{C}\left\{\frac{1}{2} \tau_{3}\left(d V \mathcal{C}_{1}+\mathcal{C}_{1} d V\right)\right\}\right. \\
& \left.+\left(\frac{1}{2} \bar{\Theta}_{C} \tau_{3} d V\right) \mathcal{C}_{1}+\bar{\Theta}_{S}\left\{\frac{1}{2}\left(d V \mathcal{S}_{1}-\mathcal{S}_{1} d V\right)\right\}\right] d \theta=\mathrm{T} d \bar{\theta} \mathcal{S}_{1} d \theta
\end{aligned}
$$

where only $d \bar{\theta}$ term in $d \bar{\Theta}_{C}$ survives to give (2.16).

\section{Hamiltonian constraints}

In this section we develop the canonical formalism and find the constraint equations for the IIB D-branes.

The lagrangian density of the system is

$$
\begin{gathered}
\mathcal{L}=\mathcal{L}^{D B I}+\mathcal{L}^{W Z} \\
\mathcal{L}^{D B I}=-\mathrm{T} \sqrt{-\operatorname{det}\left(G_{\mu \nu}+\mathcal{F}_{\mu \nu}\right)} \equiv-\mathrm{T} \sqrt{-G_{F}} .
\end{gathered}
$$

The WZ action has been determined in (2.20)

$$
\mathcal{L}^{W Z}=\left[L^{W Z}\right]_{p+1}, \quad L^{W Z}=\mathrm{T}\left(\bar{\Theta}_{C} \mathcal{S}_{1}+\bar{\Theta}_{S} \mathcal{C}_{1}\right) d \theta
$$


where [ $]_{p+1}$ means $\mathrm{p}+1$-form coefficient ( coefficient of $d \sigma^{0} \ldots d \sigma^{p}$ in the $\mathrm{p}+1$ form term ) . Let $a, b$ run from 1 to $\mathrm{p}$. We regard $\mathcal{L}$ as a function of $\Pi_{0}, \mathcal{F}_{0 a}, \dot{\theta}$ and others,

$$
\mathcal{L}=\mathcal{L}^{D B I}\left(\Pi_{0}, \mathcal{F}_{0 a}, \dot{\theta}, \ldots\right)+\mathcal{L}^{W Z}\left(\Pi_{0}, \mathcal{F}_{0 a}, \dot{\theta}, \ldots\right)
$$

We first define the canonical momenta $p_{m}, \zeta, E^{\mu}$ conjugate to $X^{m}, \theta$ and $A_{\mu}$. They are

$$
\left\{\begin{aligned}
p_{m} \equiv & \frac{\partial \mathcal{L}}{\partial \dot{x}^{m}}=\frac{\partial \mathcal{L}^{D B I}}{\partial \Pi_{0}^{m}}+\frac{\partial \mathcal{L}^{W Z}}{\partial \Pi_{0}^{m}}+\frac{\partial \mathcal{L}}{\partial \mathcal{F}_{0 a}}\left(\bar{\theta} \tau_{3} \Gamma_{m} \partial_{a} \theta\right) \\
\zeta \equiv & \frac{\partial^{r} \mathcal{L}}{\partial \dot{\theta}}=\left(\frac{\partial \mathcal{L}^{W Z}}{\partial \dot{\theta}}\right)+\left(\frac{\partial \mathcal{L}}{\partial \Pi_{0}^{m}}+\frac{\partial \mathcal{L}}{\partial \mathcal{F}_{0 a}} \frac{\partial \mathcal{F}_{0 a}}{\partial \Pi_{0}^{m}}\right)\left(-\bar{\theta} \Gamma^{m}\right) \\
& +\frac{\partial \mathcal{L}}{\partial \mathcal{F}_{0 a}}\left[-\bar{\theta} \tau_{3} \Gamma_{\ell}\left(\Pi_{a}^{\ell}+\frac{1}{2} \bar{\theta} \Gamma^{\ell} \partial_{a} \theta\right)+\frac{1}{2} \bar{\theta} \Gamma^{\ell}\left(\bar{\theta} \tau_{3} \Gamma_{\ell} \partial_{a} \theta\right)\right] \\
E^{a} \equiv & \frac{\partial \mathcal{L}}{\partial \dot{A}_{a}}=\frac{\partial \mathcal{L}}{\partial \mathcal{F}_{0 a}}=\frac{\partial \mathcal{L}^{D B I}}{\partial \mathcal{F}_{0 a}}+\frac{\partial \mathcal{L}^{W Z}}{\partial \mathcal{F}_{0 a}}
\end{aligned}\right.
$$

and

$$
E^{0} \equiv \frac{\partial \mathcal{L}}{\partial \dot{A}_{0}}=0
$$

The last one is $\mathrm{U}(1)$ constraint.

We show how the expressions for $\partial \mathcal{L}^{W Z} / \partial \prod_{0}, \partial \mathcal{L}^{W Z} / \partial \mathcal{F}_{0 a}, \partial \mathcal{L}^{W Z} / \partial \dot{\theta}$ are computed from $L^{W Z}$ without decomposing the differential forms. Using the definition (2.2) and (2.14), $\partial \mathcal{L}^{W Z} / \partial \mathcal{F}_{0 a}$ is $p+1$ form coefficient of

$$
\frac{\partial L^{W Z}}{\partial \mathcal{F}_{0 a}}=\left[d \sigma^{0} d \sigma^{a} L^{W Z}\right] .
$$

Since explicit $\Pi$ appears only in $\mathcal{S}_{1}$ and $\mathcal{C}_{1}$ in $L^{W Z}, \partial \mathcal{L}^{W Z} / \partial \Pi_{0}^{m}$ is $(\mathrm{p}+1)$ form coefficient of

$$
\begin{aligned}
\frac{\partial L^{W Z}}{\partial \Pi_{0}^{m}}=-T d \sigma^{0} & {\left[\bar{\Theta}_{C}\left(\tau_{3} \mathcal{C}_{1} \Gamma_{m}-\tau_{3} \mathcal{S}_{1} \Pi_{m}\right)\right.} \\
& \left.+\bar{\Theta}_{S}\left(\mathcal{S}_{1} \Gamma_{m}-\tau_{3} \mathcal{C}_{1} \Pi_{m}\right)\right] d \theta
\end{aligned}
$$

where we have used (A.7). $\partial \mathcal{L}^{W Z} / \partial \dot{\theta}$ is obtained from $L^{W Z}$ by using $\Delta \bar{\Theta}_{C}$ and $\Delta \bar{\Theta}_{S}$ in (A.34) and (A.35). Multiplying an odd spinor $f,\left(\partial \mathcal{L}^{W Z} / \partial \dot{\theta}\right) f$ is $(\mathrm{p}+1)$ form coefficient of

$$
\begin{aligned}
& \frac{\partial L^{W Z}}{\partial \dot{\theta}} f=T d \sigma^{0} {\left[\left(\bar{\Theta}_{C} \mathcal{S}_{1}+\bar{\Theta}_{S} \mathcal{C}_{1}\right) f\right.} \\
&\left.+\left(\bar{\Sigma}_{C}^{1}[f] \mathcal{S}_{1}+\bar{\Sigma}_{S}^{1}[f] \mathcal{C}_{1}\right) d \theta\right]
\end{aligned}
$$

where

$$
\Delta \theta=0, \quad \Delta V=V_{\theta, d \sigma^{0} f}=-d \sigma^{0} V_{\theta, f}
$$


in (A.34) and (A.35) and the explicit forms of $\bar{\Sigma}_{C}^{1}[f]$ and $\bar{\Sigma}_{S}^{1}[f]$ are given in the Appendix (A.37).

In the Hamiltonian constraints the momenta appear in the following combinations, $\tilde{E}^{a}$ and $\tilde{p}_{m}$,

$$
\left\{\begin{array}{rl}
\tilde{p}_{m} & \equiv p_{m}-\frac{\partial \mathcal{L}^{W Z}}{\partial \Pi_{0}^{m}}-E^{a}\left(\bar{\theta} \tau_{3} \Gamma_{m} \partial_{a} \theta\right) \\
\tilde{E}^{a} & \equiv E^{a}-\frac{\partial \mathcal{L}^{W Z}}{\partial \mathcal{F}_{0 a}}
\end{array} .\right.
$$

Since $\mathcal{L}^{W Z}\left(\Pi_{0}, \mathcal{F}_{0 a}, \dot{\theta}, \ldots\right)$ is linear in the velocities by construction, they are functions of canonical variables. (3.5) tells that they are contributions from the DBI action to the momenta and are

$$
\left\{\begin{array}{l}
\tilde{p}_{m}=\frac{\partial \mathcal{L}^{D B I}}{\partial \Pi_{0}^{m}}=-\frac{\mathrm{T}}{2} \sqrt{-G_{F}}\left(G_{F}^{\mu 0}+G_{F}^{0 \mu}\right) \Pi_{\mu m} \\
\tilde{E}^{a}=\frac{\partial \mathcal{L}^{D B I}}{\partial \mathcal{F}_{0 a}}=-\frac{\mathrm{T}}{2} \sqrt{-G_{F}}\left(G_{F}^{a 0}-G_{F}^{0 a}\right)
\end{array} .\right.
$$

It follows $\mathrm{p}+1$ bosonic constraints

$$
\begin{aligned}
T_{a} & \equiv \tilde{p} \Pi_{a}+\tilde{E}^{b} \mathcal{F}_{a b}=0, \quad(a=1,2, \ldots p) \\
H & \equiv \frac{1}{2}\left[\tilde{p}^{2}+\tilde{E}^{a} G_{a b} \tilde{E}^{b}+\mathrm{T}^{2} \mathbf{G}_{\mathbf{F}}\right]=0,
\end{aligned}
$$

where

$$
\mathbf{G}_{\mathbf{F}}=\operatorname{det}(G+\mathcal{F})_{a b} .
$$

The fermionic constraint follows from the definition of the momentum $\zeta$ in (3.5),

$$
\begin{aligned}
F \equiv & \zeta+p_{\ell}\left(\bar{\theta} \Gamma^{\ell}\right)+E^{a}\left[\bar{\theta} \tau_{3} \Gamma_{\ell}\left(\Pi_{a}^{\ell}+\frac{1}{2} \bar{\theta} \Gamma^{\ell} \partial_{a} \theta\right)\right. \\
& \left.-\frac{1}{2} \bar{\theta} \Gamma^{\ell}\left(\bar{\theta} \tau_{3} \Gamma_{\ell} \partial_{a} \theta\right)\right]-\left(\frac{\partial \mathcal{L}^{W Z}}{\partial \dot{\theta}}\right)=0
\end{aligned}
$$

The primary constraints are (3.13), (3.14), (3.16) and (3.6). The primitive forms of these constraint equations have been presented in [9] in connection with the discussions of the covariant quantization.

The generalized Hamiltonian $\mathcal{H}=p \dot{q}-L$ is calculated only using $(p-\partial L / \partial \dot{q})^{2} \equiv 0$ [17] as

$$
\mathcal{H}=\int d \sigma^{p}\left[\lambda^{0} H+\lambda^{a} T_{a}+F \dot{\theta}+E^{0} \dot{A}_{0}-A_{0} \partial_{a} E^{a}\right]
$$

where

$$
\lambda^{0}=\frac{-1}{\mathrm{~T} \sqrt{-G_{F}} G_{F}^{00}}, \quad \quad \lambda^{a}=\frac{G_{F}^{a 0}+G_{F}^{0 a}}{-2 G_{F}^{00}}
$$


The consistency condition that the primary $\mathrm{U}(1)$ constraint (3.6) is preserved in time requires secondary Gauss law constraint

$$
\partial_{a} E^{a}=0
$$

The consistency of other constraints requires the form of $\dot{\theta}$ in the Hamiltonian as

$$
\dot{\theta}=\left(\lambda^{0} \tilde{\tau}^{a}+\lambda^{a}\right) \partial_{a} \theta+\Xi \chi
$$

where

$$
\tilde{\tau}^{a}=\tilde{E}^{a} \tau_{3}+\mathrm{T} \tau_{3}\left[d \sigma^{a} \mathcal{C}_{1}\right]_{\mathbf{p}}
$$

and $\Xi$ is a nilpotent matrix appearing in the Poisson bracket of $F^{\prime}$ 's (see next section)

$$
\Xi \equiv \tilde{p}+\Pi_{a} \tilde{E}^{a} \tau_{3}+\mathrm{T}\left[\mathcal{S}_{1}\right]_{\mathbf{p}}
$$

and $\chi$ is an arbitrary spinor coefficient. Here $[\ldots . .]_{\mathbf{p}}$ is the spatial $\mathrm{p}$ form coefficient ( coefficient of $d \sigma^{1} \ldots d \sigma^{p}$ ) of the expression [...]. (3.20) is equivalent to the field equation of $\theta$. Using it the Hamiltonian (3.17) is written as

$$
\mathcal{H}=\int d \sigma^{p}\left[\lambda^{0} \tilde{H}+\lambda^{a} \tilde{T}_{a}+\tilde{F} \chi+E^{0} \dot{A}_{0}-A_{0} \partial_{a} E^{a}\right],
$$

where

$$
\left\{\begin{array}{l}
\tilde{H}=H+F \tilde{\tau}^{a} \partial_{a} \theta \\
\tilde{T}_{a}=T_{a}+F \partial_{a} \theta=p \partial_{a} x+\zeta \partial_{a} \theta+E^{b} F_{a b}, \\
\tilde{F}=F \Xi .
\end{array}\right.
$$

The fact that all constraints are preserved in time for arbitrary undetermined multipliers $\lambda^{\mu}, \chi, A_{0}$ and $\dot{A}_{0}$ means that the constraints $\tilde{H}, \tilde{T}_{a}, \tilde{F}, E^{0}$ and $\partial_{a} E^{a}$ are the first class constraints. $\tilde{H}$ and $\tilde{T}_{a}$ are the first class constraints generating the $p+1$ dimensional diffeomorphism. $E^{0}$ and $\partial_{a} E^{a}$ are the standard $\mathrm{U}(1)$ generators. As will be shown explicitly in the next section the fermionic constraint $F$ is a mixture of first and second class constraints as in the case of the superstring theories. The half components $\tilde{F}$ are the first class constraints generating the kappa transformations and other half are the second class ones. In the next section we find the Poisson bracket of $F$ 's and examine the kappa symmetry using the explicit form of the WZ actions (3.3).

\section{Algebra of $F^{\prime} \mathrm{s}$}

To find the (graded) Poisson bracket algebra of the fermionic constraints we define $F[f]$ by multiplying a well behaved odd function $f$

$$
\begin{aligned}
F[f] \equiv & \int d \sigma F(\sigma) f(\sigma) \\
= & \int d \sigma\left[\zeta f+p_{m}\left(\bar{\theta} \Gamma^{m} f\right)+E^{a}\left\{\bar{\theta} \tau_{3} \Gamma_{\ell} f\left(\Pi_{a}^{\ell}+\frac{1}{2} \bar{\theta} \Gamma^{\ell} \partial_{a} \theta\right)\right.\right. \\
& \left.\left.-\frac{1}{2} \bar{\theta} \Gamma^{\ell} f\left(\bar{\theta} \tau_{3} \Gamma_{\ell} \partial_{a} \theta\right)\right\}\right]+\int d \sigma\left[-\frac{\partial L^{W Z}}{\partial \dot{\theta}} f\right] \\
\equiv & F^{(1)}[f]+F^{(2)}[f]
\end{aligned}
$$


where $\frac{\partial L^{W Z}}{\partial \dot{\theta}} f$ is given in $(3.9)$ and is a function of only coordinate variables. $F^{(1)}$ is the WZ action independent part and satisfies

$$
\begin{aligned}
\left\{F^{(1)}[f], F^{(1)}[g]\right\} & =\int d \sigma\left[-2\left(\bar{f} \Gamma^{m} g\right)\left(p_{m}-E^{b} \bar{\theta} \tau_{3} \Gamma_{m} \partial_{b} \theta\right)-2\left(\bar{f} \tau_{3} \Gamma_{m} g\right)\left(E^{b} \Pi_{b}^{m}\right)\right. \\
& \left.+\frac{1}{2}\left(\partial_{a} E^{a}\right)\left\{(\bar{\theta} \Gamma f)\left(\bar{\theta} \tau_{3} \Gamma g\right)-(\bar{\theta} \Gamma g)\left(\bar{\theta} \tau_{3} \Gamma f\right)\right\}\right] .
\end{aligned}
$$

Here the last term is the Gauss law constraint term.

The WZ action dependent part $F^{(2)}$ does not contain momentum and the Poisson bracket with itself vanishes,

$$
\left\{F^{(2)}[f], F^{(2)}[g]\right\}=0
$$

while

$$
\begin{gathered}
\left\{F^{(1)}[f], F^{(2)}[g]\right\}+\left\{F^{(2)}[f], F^{(1)}[g]\right\}= \\
\int d \sigma\left[f^{t} \frac{\partial^{\ell}}{\partial \theta}\left(\frac{\partial L^{W Z}}{\partial \dot{\theta}} g\right)+\partial_{a} f^{t} \frac{\partial^{\ell}}{\partial \partial_{a} \theta}\left(\frac{\partial L^{W Z}}{\partial \dot{\theta}} g\right)-2\left(\bar{f} \Gamma^{m} \partial_{a} \theta\right) \frac{\partial^{\ell}}{\partial \Pi_{a}^{m}}\left(\frac{\partial L^{W Z}}{\partial \dot{\theta}} g\right)\right. \\
\left.-2\left(\bar{f} \tau_{3} \Gamma_{\ell} \partial_{a} \theta \Pi_{b}^{\ell}\right) \frac{\partial^{\ell}}{\partial \mathcal{F}_{a b}}\left(\frac{\partial L^{W Z}}{\partial \dot{\theta}} g\right)\right]-\{f \leftrightarrow g\},
\end{gathered}
$$

where $\{f \leftrightarrow g\}$ is terms in which $f$ and $g$ are interchanged. The sum of these terms gives the Poisson bracket of $F$ 's,

$$
\{F[f], F[g]\}=\int d \sigma\left[-2(\bar{f} \Xi g)+\frac{1}{2}\left(\partial_{a} E^{a}\right)\left\{(\bar{\theta} \Gamma f)\left(\bar{\theta} \tau_{3} \Gamma g\right)-\{f \leftrightarrow g\}\right\}\right] .
$$

The second term is the Gauss law constraint term and $\Xi$ is given by

$$
\Xi \equiv \tilde{p}+\tau_{3} \not_{a} \tilde{E}^{a}+\mathrm{T} \Xi^{(1)}, \quad \Xi^{(1)} \equiv\left[\mathcal{S}_{1}\right]_{\mathbf{p}}
$$

We can show that $\Xi$ is nilpotent on the bosonic primary constraints (3.13) and (3.14). It is reflecting the fact that the half components of the fermionic constraints are the first class while the remaining half are the second class constraints. By squaring

$$
(\Xi)^{2}=\left(\tilde{p}+\tau_{3} \Pi_{a} \tilde{E}^{a}+\mathrm{T} \Xi^{(1)}\right)^{2} .
$$

The squares of each terms are

$$
\tilde{p}^{2}=\tilde{p}^{2}, \quad\left(\tau_{3} \prod_{a} \tilde{E}^{a}\right)^{2}=\tilde{E}^{a} \tilde{E}^{b} G_{a b}, \quad\left(\Xi^{(1)}\right)^{2}=\mathbf{G}_{F} .
$$

The last equality is essentially shown in the appendix A of [5]. The sum of these three terms vanish on the bosonic primary constraint $H$ in (3.14). The cross terms are

$$
\begin{aligned}
\tilde{p}\left(\tau_{3} \Pi_{a} \tilde{E}^{a}\right)+\left(\tau_{3} \Pi_{a} \tilde{E}^{a}\right) \tilde{p} & =\tau_{3} \tilde{E}^{a}\left(2 \tilde{p} \Pi_{a}\right)=2 \tau_{3} \tilde{E}^{a} T_{a} \\
\tilde{p} \Xi^{(1)}+\Xi^{(1)} \tilde{p} & =-2\left[(\tilde{p} \Pi) \mathcal{C}_{1} \tau_{3}\right]_{\mathbf{p}}, \\
\tau_{3} \Pi_{a} \tilde{E}^{a} \Xi^{(1)}+\Xi^{(1)} \tau_{3} \Pi_{a} \tilde{E}^{a} & =-2\left[\left(\tilde{E}^{b} \mathcal{F}_{a b} d \sigma^{a}\right) \mathcal{C}_{1} \tau_{3}\right]_{\mathbf{p}} .
\end{aligned}
$$


The first one vanishes on the constraint (3.13). The sum of second and third terms also vanish on the constraint $T_{a}$. In summary

$$
(\Xi)^{2}=2 H+2 \tilde{\tau}^{a} T_{a}
$$

where $\tilde{\tau}^{a}$ is defined in (3.21). It shows the $\Xi$ is used to construct the first class constraints,

$$
\tilde{F}=F \Xi
$$

which are the generators of the kappa transformations as in the case of the superstring theories.

\section{Global SUSY transformations}

The global supersymmetry transformations of $X$ and $\theta$ are

$$
\delta_{\epsilon} \theta=\epsilon, \quad \delta_{\epsilon} X^{m}=\bar{\epsilon} \Gamma^{m} \theta .
$$

For the $\mathrm{U}(1)$ potential it is defined so that its field strength $\mathcal{F}$ is SUSY invariant [5] as

$$
\delta_{\epsilon} A=\bar{\epsilon} \tau_{3} \Gamma \theta d X-\frac{1}{6} \bar{\epsilon} \tau_{3} \bigvee \theta
$$

In the canonical formalism the supersymmetry generator is

$$
\begin{aligned}
Q \epsilon & =\int d \sigma^{p}\left(p_{m} \delta_{\epsilon} X^{m}+\zeta \delta_{\epsilon} \theta+E^{a} \delta_{\epsilon} A_{a}\right)-\int d \sigma^{p} F_{\epsilon}^{0} \\
& \equiv Q_{\epsilon}^{1}+Q_{\epsilon}^{2}
\end{aligned}
$$

$Q^{1}$ is the WZ action independent part and is

$$
\begin{aligned}
Q_{\epsilon}^{1}=\int d \sigma^{p}[\zeta \epsilon & -\bar{\theta} \Gamma^{m} \epsilon\left(p_{m}-\frac{1}{6} \bar{\theta} \tau_{3} \Gamma_{m} \partial_{a} \theta E^{a}\right) \\
& \left.-\bar{\theta} \tau_{3} \Gamma^{m} \epsilon E^{a}\left(\partial_{a} X_{m}-\frac{1}{6} \bar{\theta} \Gamma_{m} \partial_{a} \theta\right)\right] .
\end{aligned}
$$

$F_{\epsilon}^{0}$ is determined from the surface term of the SUSY variation of the lagrangian,

$$
\delta_{\epsilon} L^{W Z} \equiv d\left(\mathrm{~T}\left[q_{\epsilon}^{2}\right]\right), \quad \text { and } \quad F_{\epsilon}^{0}=\mathrm{T}\left[q_{\epsilon}^{2}\right]_{\mathbf{p}}
$$

where $\left[q_{\epsilon}^{2}\right]_{\mathbf{p}}$ is spatial p-form coefficient of $\left[q_{\epsilon}^{2}\right]$ and

$$
Q_{\epsilon}^{2}=-\mathrm{T} \int d \sigma^{p}\left[q_{\epsilon}^{2}\right]_{\mathbf{p}}
$$

In the WZ action (2.20) $\mathcal{S}_{1}, \mathcal{C}_{1}$ and $d \theta$ are SUSY invariant only $\bar{\Theta}_{C}$ and $\bar{\Theta}_{S}$ are varied

$$
\delta_{\epsilon} L^{W Z}=\mathrm{T}\left(\delta_{\epsilon} \bar{\Theta}_{C} \mathcal{S}_{1}+\delta_{\epsilon} \bar{\Theta}_{S} \mathcal{C}_{1}\right) d \theta=\bar{\Psi}_{S} d \theta
$$

where we define

$$
\bar{\Psi}_{S} \equiv \mathrm{T}\left(\delta_{\epsilon} \bar{\Theta}_{C} \mathcal{S}_{1}+\delta_{\epsilon} \bar{\Theta}_{S} \mathcal{C}_{1}\right), \quad \bar{\Psi}_{C} \equiv \mathrm{T}\left(-\delta_{\epsilon} \bar{\Theta}_{C} \mathcal{C}_{1} \tau_{3}+\delta_{\epsilon} \bar{\Theta}_{S} \mathcal{S}_{1}\right)
$$


Using (2.13) and (2.15) they satisfy

$$
d \bar{\Psi}_{S}=-\frac{1}{2} \bar{\Psi}_{C} d V, \quad d \bar{\Psi}_{C}=-\frac{1}{2} \bar{\Psi}_{S} \tau_{3} d V .
$$

Writing (5.7) as

$$
\delta_{\epsilon} L^{W Z}=d\left[\bar{\Psi}_{S} \theta\right]-\left(d \bar{\Psi}_{S}\right) \theta
$$

and use (5.9) and (2.11). As in the procedure leading to (2.20) we continue to arrive at

$$
\delta L^{W Z}=d\left[\bar{\Psi}_{S} \Theta_{C}+\bar{\Psi}_{C} \tau_{3} \Theta_{S}\right] .
$$

Up to now we have only used the fact that $\delta_{\epsilon}$ is even variation and the transformation is global, $\delta_{\epsilon} d \theta=0$. The forms of SUSY variation $\delta_{\epsilon} \bar{\Theta}_{C}$ and $\delta_{\epsilon} \bar{\Theta}_{S}$ are

$$
\delta_{\epsilon} \bar{\Theta}_{C}=\bar{\epsilon}+d \bar{\Sigma}_{C}^{1}[\epsilon]+\bar{\Sigma}_{C}^{2}[\epsilon], \quad \delta_{\epsilon} \bar{\Theta}_{S}=d \bar{\Sigma}_{S}^{1}[\epsilon]+\bar{\Sigma}_{S}^{2}[\epsilon],
$$

where $\bar{\Sigma}_{C}^{1}[\epsilon]$ and $\bar{\Sigma}_{S}^{1}[\epsilon]$ are given in the Appendix (A.37) and $\bar{\Sigma}_{C}^{2}[\epsilon]$ and $\bar{\Sigma}_{S}^{2}[\epsilon]$ are satisfying

$$
\begin{aligned}
& \bar{\Sigma}_{C}^{2}[\epsilon]=-\frac{1}{2} \bar{\Sigma}_{S}^{1}[\epsilon] d V-\bar{\Theta}_{S} V_{\epsilon, d \theta}, \\
& \bar{\Sigma}_{S}^{2}[\epsilon]=\left(\frac{1}{2} \bar{\Sigma}_{C}^{1}[\epsilon] d V-\bar{\Theta}_{C} V_{\epsilon, d \theta}\right) \tau_{3} .
\end{aligned}
$$

Using them we can determine the surface term appearing in the SUSY variation of the WZ lagrangian,

$$
\begin{aligned}
\delta_{\epsilon} L^{W Z}=\mathrm{T} d & {\left[\bar{\epsilon}\left(\mathcal{S}_{1} \Theta_{C}-\mathcal{C}_{1} \Theta_{S}\right)+\left(\bar{\Sigma}_{C}^{1}[\epsilon] \mathcal{S}_{1}+\bar{\Sigma}_{S}^{1}[\epsilon] \mathcal{C}_{1}\right) d \theta\right.} \\
& -\bar{\Theta}_{S} V_{\epsilon, d \theta} \mathcal{S}_{1} \Theta_{C}+\bar{\Theta}_{S} V_{\epsilon, d \theta} \mathcal{C}_{1} \Theta_{S} \\
& \left.+\bar{\Theta}_{C} V_{\epsilon, d \theta} \mathcal{S}_{1} \Theta_{S}+\bar{\Theta}_{C} V_{\epsilon, d \theta} \mathcal{C}_{1} \tau_{3} \Theta_{C}\right] \equiv \mathrm{T} d\left[q_{\epsilon}^{2}\right] .
\end{aligned}
$$

It gives $\left[q_{\epsilon}^{2}\right]$ and $Q_{\epsilon}^{2}$ is determined from (5.6).

\section{SUSY algebra and Central charges}

As was discussed in [13] [11] the SUSY algebras of extended objects can induce central extensions by the pseudo-invariance of the WZ action under the SUSY transformations. We discuss it in the present case of D-p-branes.

We have determined the supersymmetry charge $Q=Q^{1}+Q^{2}$ for general IIB D-branes in (5.4) and (5.6) with $q_{\epsilon}^{2}$ given in (5.14), up to an exact form, as

$$
\begin{aligned}
q_{\epsilon}^{2} & =\bar{\epsilon}\left(\mathcal{S}_{1} \Theta_{C}-\mathcal{C}_{1} \Theta_{S}\right)+\left(\bar{\Sigma}_{C}^{1}[\epsilon] \mathcal{S}_{1}+\bar{\Sigma}_{S}^{1}[\epsilon] \mathcal{C}_{1}\right) d \theta \\
& -\bar{\Theta}_{S} V_{\epsilon, d \theta} \mathcal{S}_{1} \Theta_{C}+\bar{\Theta}_{S} V_{\epsilon, d \theta} \mathcal{C}_{1} \Theta_{S} \\
& +\bar{\Theta}_{C} V_{\epsilon, d \theta} \mathcal{S}_{1} \Theta_{S}+\bar{\Theta}_{C} V_{\epsilon, d \theta} \mathcal{C}_{1} \tau_{3} \Theta_{C} .
\end{aligned}
$$

In this section we will find the SUSY algebra and determine the central charges in terms of the dynamical variables $X^{m}, \theta$ and $A_{\mu}$. 
In the (graded) Poisson bracket of the supersymmetry charges, $\left\{Q_{\epsilon}^{1}, Q_{\epsilon^{\prime}}^{1}\right\}$ is independent of the WZ action and is common for all IIB D-branes,

$$
\begin{aligned}
\left\{Q_{\epsilon}^{1}, Q_{\epsilon^{\prime}}^{1}\right\} & =\int d \sigma^{p}\left[2\left(\bar{\epsilon} \Gamma \epsilon^{\prime}\right) p+2\left(\bar{\epsilon} \tau_{3} \Gamma \epsilon^{\prime}\right) \partial_{a} X E^{a}\right. \\
& \left.-\frac{1}{2}\left(\partial_{a} E^{a}\right)\left(\bar{\theta} \tau_{3} \Gamma \epsilon \bar{\theta} \Gamma \epsilon^{\prime}-\bar{\theta} \tau_{3} \Gamma \epsilon^{\prime} \bar{\theta} \Gamma \epsilon\right)\right] .
\end{aligned}
$$

The first term is the total momentum term of the SUSY algebra. The last term is the Gauss law constraint term. Since $Q^{1}$ generates SUSY transformations for coordinate variables and $Q^{2}$ contains only coordinates

$$
\begin{gathered}
\left\{Q_{\epsilon}^{1}, Q_{\epsilon^{\prime}}^{2}\right\}+\left\{Q_{\epsilon}^{2}, Q_{\epsilon^{\prime}}^{1}\right\}=-\delta_{\epsilon} Q_{\epsilon^{\prime}}^{2}+\delta_{\epsilon^{\prime}} Q_{\epsilon}^{2} \equiv \delta_{\left[\epsilon^{\prime}\right.} Q_{\epsilon]}^{2}, \\
\left\{Q_{\epsilon}^{2}, Q_{\epsilon^{\prime}}^{2}\right\}=0,
\end{gathered}
$$

where our anti-symmetrization convention is $A_{[\epsilon} B_{\left.\epsilon^{\prime}\right]}=A_{\epsilon} B_{\epsilon^{\prime}}-A_{\epsilon^{\prime}} B_{\epsilon}$. Thus the SUSY algebra becomes

$$
\begin{aligned}
\left\{Q_{\epsilon}, Q_{\epsilon^{\prime}}\right\} & =\int d \sigma^{p}\left[2\left(\bar{\epsilon} \Gamma \epsilon^{\prime}\right) p+2\left(\bar{\epsilon} \tau_{3} \Gamma \epsilon^{\prime}\right) \partial_{a} X E^{a}\right. \\
& \left.-\frac{1}{2}\left(\partial_{a} E^{a}\right)\left(\bar{\theta} \tau_{3} \Gamma \epsilon \bar{\theta} \Gamma \epsilon^{\prime}-\bar{\theta} \tau_{3} \Gamma \epsilon^{\prime} \bar{\theta} \Gamma \epsilon\right)\right] \\
& \left.-\mathrm{T} \int d \sigma^{p}\left[\delta_{\left[\epsilon^{\prime}\right.} q_{\epsilon}^{2}\right]\right]_{\mathbf{p}} .
\end{aligned}
$$

We calculate the last term in the differential form $\delta_{\left[\epsilon^{\prime}\right.} q_{\epsilon]}^{2}$. Since $d \theta, \Pi, \mathcal{F}$ are SUSY invariant, only the explicit $\theta$ 's are varied in $q_{\epsilon}^{2}$. We use (5.12) and (5.13) for $\delta_{\epsilon} \bar{\Theta}_{C}$ and $\delta_{\epsilon} \bar{\Theta}_{S}$ and

$$
\delta_{\left[\epsilon^{\prime}\right.}\left(\bar{\Sigma}_{C}^{1}\left[\epsilon_{]}\right]\right) \equiv d \Lambda_{C}^{1}+\Lambda_{C}^{2}, \quad \delta_{\left[\epsilon^{\prime}\right.}\left(\bar{\Sigma}_{S}^{1}\left[\epsilon_{]}\right]\right) \equiv d \Lambda_{S}^{1}+\Lambda_{S}^{2}
$$

where the explicit form of $\Lambda^{1}$ 's are given in Appendix (A.40). $\Lambda^{2}$ 's are related to $\Lambda^{1}$ 's as

$$
\begin{aligned}
\Lambda_{S}^{2} & =-\frac{1}{2} \Lambda_{C}^{1} \tau_{3} d V-\bar{\Theta}_{C} \tau_{3} V_{\epsilon^{\prime}, \epsilon}-\bar{\Sigma}_{C}^{1}\left[\left[\epsilon^{\prime}\right] \tau_{3} V_{\epsilon], d \theta}\right. \\
\Lambda_{C}^{2} & =\frac{1}{2} \Lambda_{S}^{1} d V+\bar{\Theta}_{S} V_{\epsilon^{\prime}, \epsilon}-\bar{\Sigma}_{S}^{1}\left[\left[\epsilon^{\prime}\right] V_{\epsilon], d \theta} .\right.
\end{aligned}
$$

After some manipulation we obtain, up to an exact form,

$$
\begin{aligned}
\delta_{\left[\epsilon^{\prime}\right.} q_{\epsilon]}^{2}= & 2\left[\left(\bar{\epsilon}-\bar{\Theta}_{S} V_{\epsilon, d \theta}\right) \mathcal{S}_{1}\left(\epsilon^{\prime}+V_{\epsilon^{\prime}, d \theta} \Theta_{S}\right)\right. \\
& +\left(\bar{\epsilon}-\bar{\Theta}_{S} V_{\epsilon, d \theta}\right) \mathcal{C}_{1} \tau_{3} V_{\epsilon^{\prime}, d \theta} \Theta_{C}+\bar{\Theta}_{C} V_{\epsilon, d \theta} \mathcal{C}_{1} \tau_{3}\left(\epsilon^{\prime}+V_{\epsilon^{\prime}, d \theta} \Theta_{S}\right) \\
& \left.-\bar{\Theta}_{C} V_{\epsilon, d \theta} \mathcal{S}_{1} \tau_{3} V_{\epsilon^{\prime}, d \theta} \Theta_{C}\right] .
\end{aligned}
$$

We will express the SUSY algebra in the standard form,

$$
\left\{Q_{\alpha A}, Q_{\beta B}\right\}=-2\left(C \Gamma_{m}\right)_{\alpha \beta} \delta_{A B} \mathcal{P}^{m}+\left(C \Gamma_{M}\right)_{\alpha \beta}\left(\tau_{J}\right)_{A B} Z_{J}^{M}
$$


where the total momentum term is separated as usual and $\left(C \Gamma_{M} \tau_{J}\right)$ is the complete basis of gamma matrices and tau matrices [1]. $J$ runs from $0,1,2$ and 3,

$$
\operatorname{tr} \frac{1}{2}\left(\tau_{J} \tau_{L}\right)=\delta_{J L}, \quad(J, L=0,1,2,3)
$$

$\Gamma_{M}$ 's are vectors $\Gamma_{m}$, totally anti-symmetric rank three tensors $\Gamma_{m_{1} m_{2} m_{3}}$ and totally antisymmetric (anti-) self-dual rank five tensors $\Gamma_{m_{1} m_{2} m_{3} m_{4} m_{5}}$. They are $10+120+252 / 2=16^{2}$ orthonormal basis for gamma matrices with the definite Weyl projection, (the projection operators are abbreviated throughout),

$$
\operatorname{tr} \frac{1}{2^{4}}\left(\left(\Gamma^{N} C^{-1}\right)\left(C \Gamma_{M}\right)\right)=\delta_{M}^{N}
$$

where $\operatorname{tr} 1=2^{4}$ in the subspace by the Weyl projection. We reverse the order of tensor indices for the $\Gamma^{M}$. For example

$$
\operatorname{tr} \frac{1}{2^{4}}\left(\left(\Gamma^{n_{3} n_{2} n_{1}} C^{-1}\right)\left(C \Gamma_{m_{1} m_{2} m_{3}}\right)\right)=\left(\delta_{m_{1}}^{n_{1}} \delta_{m_{2}}^{n_{2}} \delta_{m_{3}}^{n_{3}}+\ldots\right)
$$

where ... means anti-symmetrized terms.

Using (6.5) we obtain the SUSY algebra

$$
\begin{aligned}
\left\{Q_{\alpha A}, Q_{\beta B}\right\} & =-2\left(C \Gamma_{m}\right)_{\alpha \beta} \delta_{A B} \mathcal{P}^{m} \\
& -2\left(\tau_{3}\right)_{A B}\left(C \Gamma_{m}\right)_{\alpha \beta} \int d \sigma^{p}\left(\partial_{a} X E^{a}\right) \\
& +\frac{1}{4}\left(\tau_{1}\right)_{A B}\left(C \Gamma_{m}\right)_{\alpha \beta} \int d \sigma^{p}\left(\partial_{a} E^{a}\right) \bar{\theta} \Gamma^{m}\left(i \tau_{2}\right) \theta \\
& -\frac{1}{8}\left(i \tau_{2}\right)_{A B}\left(C \Gamma_{m_{1} m_{2} m_{3}}\right)_{\alpha \beta} \int d \sigma^{p}\left(\partial_{a} E^{a}\right) \bar{\theta} \Gamma^{m_{3} m_{2} m_{1}} \tau_{1} \theta \\
& -\left(\tilde{\tau}_{J}\right)_{A B}\left(C \Gamma_{M}\right)_{\alpha \beta} \frac{2 \mathrm{~T}}{2^{5}}\left(1+(-)^{\sigma_{M}+J}\right) \int d \sigma^{p}\left[U_{J}^{M}\right]_{\mathbf{p}} .
\end{aligned}
$$

The first term is the total momentum term. The second term is diagonal $\tau_{3}$ term giving $Z_{3}^{m}$,

$$
Z_{3}^{m}=-2 \int d \sigma^{p}\left(\partial_{a} X E^{a}\right)
$$

Regarding the Gauss law constraint (3.19) it is a topological charge. The third and forth terms are the Gauss law constraint terms of (6.5) and vanish on shell. (The sum of $m_{1} m_{2} m_{3}$ is only over independent anti-symmetric indices.) The last term is the contribution from the WZ action, i.e. from (6.8) and depends on the form of the WZ action explicitly. Here $\tilde{\tau}_{J}=\tau_{J}$ for $J=1,2$ and $\tilde{\tau}_{J}=0$ otherwise. $\sigma_{M}=1$ for $M=m$ and $M=m_{1} m_{2} m_{3} m_{4} m_{5}$, and $\sigma_{M}=0$ for $M=m_{1} m_{2} m_{3}$. It shows it contributes only to off diagonal $\left\{Q_{1}, Q_{2}\right\}$ and the vectors and the selfdual totally antisymmetric rank 5 tensors associate to $\tau_{1}$ and the totally antisymmetric rank 3 tensors associate to $\tau_{2}$. It 
is consistent with the fact that $(6.9)$ is symmetric under simultaneous exchange of $(\alpha A)$ and $(\beta B)$. The possible central charges are (6.14) and

$$
\begin{aligned}
Z_{1}^{m} & =-\frac{\mathrm{T}}{8} \int d \sigma^{p}\left[U_{1}^{m}\right]_{\mathbf{p}}-\frac{1}{4} \int d \sigma^{p}\left(\partial_{a} E^{a}\right) \bar{\theta} \Gamma^{m} \tau_{1} \tau_{3} \theta, \\
Z_{2}^{m_{3} m_{2} m_{1}} & =-\frac{\mathrm{T}}{8} \int d \sigma^{p}\left[U_{2}^{m_{3} m_{2} m_{1}}\right]_{\mathbf{p}}-\frac{1}{8} \int d \sigma^{p}\left(\partial_{a} E^{a}\right) \bar{\theta} \Gamma^{m_{3} m_{2} m_{1}} \tau_{2} \tau_{3} \theta . \\
Z_{1}^{m_{5} m_{4} m_{3} m_{2} m_{1}} & =-\frac{\mathrm{T}}{8} \int d \sigma^{p}\left[U_{1}^{m_{5} m_{4} m_{3} m_{2} m_{1}}\right]_{\mathbf{p}} .
\end{aligned}
$$

where U's are given from (6.8) as

$$
\begin{aligned}
U_{J}^{M}= & \frac{1}{2} \operatorname{Tr}\left(\tilde{\tau}_{J} \Gamma^{M} \mathcal{S}_{1}\right) \\
+ & 2\left(d \bar{\theta} \tilde{\tau}_{J} \Gamma_{\ell} \Gamma^{M} \mathcal{S}_{1} \Gamma^{\ell} \Theta_{S}+d \bar{\theta} \tilde{\tau}_{J} \tau_{3} \Gamma_{\ell} \Gamma^{M} \mathcal{C}_{1} \Gamma^{\ell} \Theta_{C}\right) \\
- & \left(d \bar{\theta} \Gamma^{\ell} \Gamma^{M} \Gamma^{n} \tilde{\tau}_{J} d \theta\right)\left(\bar{\Theta}_{S} \Gamma_{n} \mathcal{S}_{1} \Gamma_{\ell} \Theta_{S}+\bar{\Theta}_{C} \Gamma_{n} \mathcal{S}_{1} \Gamma_{\ell} \tau_{3} \Theta_{C}+\right. \\
& \left.\quad+\bar{\Theta}_{C} \Gamma_{n} \mathcal{C}_{1} \Gamma_{\ell} \tau_{3} \Theta_{S}-\bar{\Theta}_{S} \Gamma_{n} \mathcal{C}_{1} \Gamma_{\ell} \tau_{3} \Theta_{C}\right) \\
+ & \left(d \bar{\theta} \Gamma^{\ell} \Gamma^{M} \Gamma^{n} \tilde{\tau}_{J} \tau_{3} d \theta\right)\left(\bar{\Theta}_{S} \Gamma_{n} \mathcal{S}_{1} \Gamma_{\ell} \tau_{3} \Theta_{S}+\bar{\Theta}_{C} \Gamma_{n} \mathcal{S}_{1} \Gamma_{\ell} \Theta_{C}\right. \\
& \left.+\bar{\Theta}_{C} \Gamma_{n} \mathcal{C}_{1} \Gamma_{\ell} \Theta_{S}-\bar{\Theta}_{S} \Gamma_{n} \mathcal{C}_{1} \Gamma_{\ell} \Theta_{C}\right) .
\end{aligned}
$$

These Z's are surface terms. It is seen from the fact that (6.8) is a closed form

$$
d\left(\delta_{\left[\epsilon^{\prime}\right.} q_{\epsilon]}^{2}\right)=0 .
$$

The boundary conditions of the D-branes are chosen so that there is no supercurrent flows from the boundaries or they are periodic for the compact configurations. The surface terms give topological charges, which may appear from the non-trivial configurations of the coordinates $X^{m}$ and the gauge fields $A_{\mu}$. Assuming the suitable boundary conditions for $\theta, \Pi$ and $\mathcal{F}$ the possible topological terms arise only from the $\bar{\epsilon} \mathcal{S}_{1} \epsilon$ term in (6.8) and $U_{J}^{M}$ in the SUSY algebra (6.16) becomes

$$
U_{J}^{M}=\frac{1}{2} \operatorname{Tr}\left(\tilde{\tau}_{J} \Gamma^{M} \mathcal{S}_{B}(d X) \tau_{1}\right) e^{d A} .
$$

where the definition of $\mathcal{S}_{B}(d X)$ is given from (2.5). The expression of the topological central charges $Z_{1}$ and $Z_{2}$ of (6.9), up to the Gauss law constraint, are listed in table 1 .

In table 1 , for example in $Z_{2}^{(3)},(d X)^{3}$ is a symbolic notation of $d X^{m_{1}} d X^{m_{2}} d X^{m_{3}}$ and ${ }^{*}(d X)^{7}$ is $\epsilon^{m_{1} m_{2} m_{3} \ell_{1} \ell_{2} \ell_{3} \ell_{4} \ell_{5} \ell_{6} \ell_{7}} d X_{\ell_{1}} d X_{\ell_{2}} d X_{\ell_{3}} d X_{\ell_{4}} d X_{\ell_{5}} d X_{\ell_{6}} d X_{\ell_{7}} / 7$ !.

\section{Summary and Discussions}

In this paper we develop the canonical formulation of general IIB D-p-branes as the first step towards the canonical quantization. In order to perform it we first found the explicit form of WZ actions for IIB D-branes. The differential equation determining 
Table 1: SUSY central charges, $Z_{1}$ and $Z_{2}$

\begin{tabular}{|c|c|c|c|}
\hline & $Z_{1}^{(1)}$ & $Z_{1}^{(5)}$ & $Z_{2}^{(3)} / i$ \\
\hline D-1 & $d X$ & 0 & 0 \\
\hline D-3 & $d X d A$ & 0 & $(d X)^{3}$ \\
\hline D-5 & $d X(d A)^{2} / 2 !$ & $\left((d X)^{5}+^{*}(d X)^{5}\right) / 2$ & $(d X)^{3} d A$ \\
\hline D-7 & $d X(d A)^{3} / 3 !$ & $\left((d X)^{5}+^{*}(d X)^{5}\right) / 2(d A)$ & $(d X)^{3}(d A)^{2} / 2 !++^{*}(d X)^{7}$ \\
\hline D-9 & $d X(d A)^{4} / 4 !+^{*}(d X)^{9}$ & $\left((d X)^{5}+^{*}(d X)^{5}\right)(d A)^{2} / 4$ & $(d X)^{3}(d A)^{3} / 3 !++^{*}(d X)^{7} d A$ \\
\hline
\end{tabular}

the general WZ action given in (1.1) is integrated by utilizing differential forms. We found convenient spinor forms $\Theta_{C}$ and $\Theta_{S}$ satisfying (2.13). In terms of $\Theta_{C}$ and $\Theta_{S}$, in addition to $\mathcal{S}_{B}$ and $\mathcal{C}_{B}$, we can handle formulas for D-p-branes in the differential forms. Especially the WZ action is expressed in the simple form as in (2.20). We determined the set of constraint equations completely in the Hamiltonian formalism. The algebra of the fermionic constraints tells that a half of them are first class constraints generating the kappa symmetry and another half are second class constraints.

We also constructed SUSY charges and computed Poisson bracket algebra. We found expressions of SUSY central charges in terms of worldvolume variables. The appearance of the topological charges in the SUSY algebra of extended models have been discussed in 13] 11] [18]. Recently Hammer examined the SUSY algebra of IIA D-p-branes [19]. The possible forms of the conserved SUSY charges and their algebra have been discussed mainly from the point of view of the algebraic consistency. Although the full WZ action is not taken into account topological charges are found using the leading terms. They correspond, in our discussions of IIB theory, to $\theta$ independent part of $U^{1}$ term (6.18). The possible interpretations of the central charges are also presented in them.

The case for type IIA D-branes can be done straightforwardly. As is suggested [5] the $\tau_{3}$ matrix is replaced by $\Gamma_{11}$ in appropriate ways for the IIA models. However we didn't go into it since it requires some more delicate notations. We leave the IIA theories for future publication [20].

Interesting issue is the covariant quantization of the kappa invariant theories, the superstrings and super D-branes. Kallosh [9] discussed it for the D0 brane in detail and drew sketches for D-p-branes. We discussed it for the D1 brane in detail in [10] and pointed out origins of its difficulties. Towards the covariant quantization of super-Dbranes it requires further investigations.

\section{Acknowledgements}

The authors would like to thank Joaquim Gomis, Yuji Igarashi and Katsumi Itoh for helpful discussions. M.H. is partially supported by the Sasakawa Scientific Research Grant from the Japan Science Society. 


\section{A Definitions and useful formulas}

The definition of $\mathcal{C}_{B}$ and $\mathcal{S}_{B}$ are same as in [5] ( their $\psi$ is denoted as $\left.\not / \not\right)$

$$
\begin{aligned}
& \mathcal{C}_{B}(\mathrm{\Pi})=\sum_{\ell=0} \tau_{3}^{\ell+1} \frac{\Pi^{2 \ell}}{(2 \ell) !}=\tau_{3}+\frac{\Pi^{2}}{2 !}+\tau_{3} \frac{\Pi^{4}}{4 !}+\ldots, \\
& \mathcal{S}_{B}(\not \mathbb{L})=\sum_{\ell=0} \tau_{3}^{\ell} \frac{\Pi^{2 \ell+1}}{(2 \ell+1) !}=\not \Lambda+\tau_{3} \frac{\Pi^{3}}{3 !}+\frac{\Pi^{5}}{5 !}+\ldots .
\end{aligned}
$$

For any one form vector such as $\Pi^{m}$ we have

$$
\Gamma_{m} \mathbb{Z}^{s}=(-)^{s} \mathbb{Z}^{s} \Gamma_{m}+2 s \Pi_{m} \Pi^{s-1}
$$

then

$$
\begin{aligned}
& \mathcal{S}_{B} \Gamma_{m}=-\Gamma_{m} \mathcal{S}_{B}+2 \tau_{3} \mathcal{C}_{B} \Pi_{m}, \\
& \mathcal{C}_{B} \Gamma_{m}=\Gamma_{m} \mathcal{C}_{B}+2 \mathcal{S}_{B} \Pi_{m}
\end{aligned}
$$

For odd variation $\delta$ and for any one form vector such as $\Pi^{m}$

$$
\delta \Pi^{\ell}=\ell(\ell-1)(-)^{\ell} \Pi^{\ell-2}\left(\Pi_{m} \delta \Pi^{m}\right)-(-)^{\ell} \ell \Pi^{\ell-1}\left(\Gamma_{m} \delta \Pi^{m}\right) .
$$

Using them we have

$$
\begin{aligned}
\delta \mathcal{S}_{B} & =\tau_{3} \mathcal{C}_{B}\left(\Gamma_{m} \delta \Pi^{m}\right)-\tau_{3} \mathcal{S}_{B}\left(\Pi_{m} \delta \Pi^{m}\right) \\
\delta \mathcal{C}_{B} & =-\mathcal{S}_{B}\left(\Gamma_{m} \delta \Pi^{m}\right)+\tau_{3} \mathcal{C}_{B}\left(\Pi_{m} \delta \Pi^{m}\right)
\end{aligned}
$$

The formula for even variation $\Delta$ are obtained by writing $\delta=c \Delta$ and removing the odd constant $c$ from the both sides. From (A.4)

$$
\Delta \Pi^{\ell}=-\ell(\ell-1) \Pi^{\ell-2}\left(\Pi_{m} \Delta \Pi^{m}\right)+\ell \Pi^{\ell-1}\left(\Gamma_{m} \Delta \Pi^{m}\right) .
$$

From (A.5) we have

$$
\begin{aligned}
\Delta \mathcal{S}_{B} & =\tau_{3} \mathcal{C}_{B}\left(\Gamma_{m} \Delta \Pi^{m}\right)-\tau_{3} \mathcal{S}_{B}\left(\Pi_{m} \Delta \Pi^{m}\right) \\
\Delta \mathcal{C}_{B} & =+\mathcal{S}_{B}\left(\Gamma_{m} \Delta \Pi^{m}\right)-\tau_{3} \mathcal{C}_{B}\left(\Pi_{m} \Delta \Pi^{m}\right)
\end{aligned}
$$

For two form $\mathcal{F}$

$$
\delta e^{\mathcal{F}}=e^{\mathcal{F}} \delta \mathcal{F}, \quad \Delta e^{\mathcal{F}}=e^{\mathcal{F}} \Delta \mathcal{F}
$$

Up to now we have used only properties of gamma matrices. 
In this paper we consider three kind of derivations, exterior derivative, global SUSY transformations and kappa transformations; $\delta \rightarrow d, c \delta_{\epsilon}, c \delta_{\kappa}$, where $\mathrm{c}$ is an odd constant. Under these derivations

$$
\begin{array}{lll}
d \theta, & d \Pi^{m}=d \bar{\theta} \Gamma^{m} d \theta, & d \mathcal{F}=d \bar{\theta} \tau_{3} \not \Lambda d \theta, \\
\delta \theta=c \epsilon, & \delta \Pi^{m}=0, & \delta \mathcal{F}=0, \\
\delta \theta=c \delta_{\kappa} \theta, & \delta \Pi^{m}=2 \delta \bar{\theta} \Gamma^{m} d \theta, & \delta \mathcal{F}=2 \delta \bar{\theta} \tau_{3} \not \Lambda d \theta,
\end{array}
$$

respectively. We can express them collectively as

$$
\delta_{\chi} \Pi^{m}=\bar{\chi} \Gamma^{m} d \theta, \quad \delta_{\chi} \mathcal{F}=\bar{\chi} \tau_{3} \not \Lambda d \theta
$$

and

$$
\begin{aligned}
& \chi=d \theta \quad \text { for } \quad d, \\
& \chi=0 \text { for SUSY, } \\
& \chi=2 \delta \theta \text { for kappa. }
\end{aligned}
$$

Using the notation (2.6), $\mathcal{S}_{1}$ and $\mathcal{C}_{1}$ in (2.14) satisfy

$$
\begin{aligned}
& \delta_{\chi} \mathcal{S}_{1}=\frac{1}{2} \tau_{3}\left[V_{\chi, d \theta} \mathcal{C}_{1}+\mathcal{C}_{1} \bigvee_{\chi, d \theta}\right] \\
& \delta_{\chi} \mathcal{C}_{1}=\frac{1}{2}\left[V_{\chi, d \theta} \mathcal{S}_{1}-\mathcal{S}_{1} V_{\chi, d \theta}\right] .
\end{aligned}
$$

Especially for $\delta=d$ it gives (2.15).

Another important quantities we use are $\mathrm{j}$-form spinor $\Theta_{j}$ (with parity $\mathrm{j}+1$ ) defined by

$$
\Theta_{j}=V \Theta_{j-1}=\ldots=V^{j} \Theta_{0}, \quad \Theta_{0} \equiv \theta .
$$

It follows

$$
\bar{\Theta}_{j}=(-)^{j-1} \bar{\Theta}_{j-1} \bigvee=\ldots=(-)^{\frac{j(j-1)}{2}} \bar{\theta} \bigvee^{j} .
$$

We further define

$$
\begin{aligned}
\Theta_{C} & =\sum_{n=0} \frac{1}{(4 n+1) ! !}\left(-\tau_{3}\right)^{n} \Theta_{2 n}=\sum_{n=0} \frac{1}{(4 n+1) ! !}\left(-\tau_{3}\right)^{n} V^{2 n} \theta \\
\Theta_{S} & \left.=\sum_{n=0} \frac{1}{(4 n+3) ! !}\left(-\tau_{3}\right)^{n+1} \Theta_{2 n+1}=\sum_{n=0} \frac{1}{(4 n+3) ! !}\left(-\tau_{3}\right)^{n+1} V^{2 n+1} \theta, \text { (A. } 20\right)
\end{aligned}
$$


or equivalently

$$
\begin{aligned}
\bar{\Theta}_{C} & =\sum_{n=0} \frac{1}{(4 n+1) ! !} \bar{\Theta}_{2 n}\left(-\tau_{3}\right)^{n}=\sum_{n=0} \frac{1}{(4 n+1) ! !} \bar{\theta} V^{2 n} \tau_{3}^{n}, \\
\bar{\Theta}_{S} & =\sum_{n=0} \frac{1}{(4 n+3) ! !} \bar{\Theta}_{2 n+1}\left(-\tau_{3}\right)^{n+1}=-\sum_{n=0} \frac{1}{(4 n+3) ! !} \bar{\theta} V^{2 n+1} \tau_{3}{ }^{n+1} .
\end{aligned}
$$

We start with finding formulas of variations of $\Theta$ 's under general odd derivations of $\theta$.

$$
\begin{aligned}
\delta \bar{\Theta}_{j}= & -\frac{2 j+1}{2} \bar{\Theta}_{j-1} \delta V \\
& +(-)^{j}(j+1)(j-1) \bar{\Theta}_{j-2}(\delta B B)-(-)^{\frac{j(j-1)}{2}} \bar{\rho}_{\delta} \bigvee^{j-1}
\end{aligned}
$$

where

$$
\bar{\rho}_{\delta} \equiv \frac{1}{2} \bar{\theta} d \bigvee_{\theta, \delta \theta}+d \bar{\theta} \bigvee_{\theta, \delta \theta}=-\frac{1}{2} \bar{\theta}(\delta \bigvee)-(\delta \bar{\theta}) \bigvee
$$

(A.22) is valid also for $\mathrm{j}=1$ since the second term vanish and is proved by the mathematical induction.

Using it we have

$$
\begin{aligned}
\delta \bar{\Theta}_{C} & =\delta \bar{\theta}-\frac{1}{2} \bar{\Theta}_{S} \delta \bigvee \\
& +\sum_{n=1} \frac{4 n^{2}-1}{(4 n+1) ! !} \bar{\Theta}_{2 n-2}\left(-\tau_{3}\right)^{n}(\delta V V)-\sum_{n=1} \frac{1}{(4 n+1) ! !} \bar{\rho}_{\delta} W^{2 n-1} \tau_{3}{ }^{n}
\end{aligned}
$$

and

$$
\begin{aligned}
\delta \bar{\Theta}_{S} & =\frac{1}{2} \bar{\Theta}_{C} \tau_{3} \delta V \\
& -\sum_{n=1} \frac{2 n(2 n+2)}{(4 n+3) ! !} \bar{\Theta}_{2 n-1}\left(-\tau_{3}\right)^{n+1}(\delta V V)+\sum_{n=0} \frac{1}{(4 n+3) ! !} \bar{\rho}_{\delta} V^{2 n} \tau_{3}{ }^{n+1}
\end{aligned}
$$

For $\delta=d,(\mathrm{~A} .22)$ becomes

$$
\begin{aligned}
& d \bar{\Theta}_{j}=-\frac{2 j+1}{2} \bar{\Theta}_{j-1} d V, \\
& d \Theta_{j}=\frac{2 j+1}{2} d V \Theta_{j-1}, \quad(j=1,2, \ldots)
\end{aligned}
$$

since the second and third terms of (A.22) disappear by

$$
(d V V)=0, \quad \rho=\left(\frac{1}{2} \bar{\theta} d V+d \bar{\theta} \bigvee\right)=0 .
$$

Similarly (A.24) becomes for $\delta=d$

$$
\begin{array}{rlrl}
d \bar{\Theta}_{C} & =d \bar{\theta}-\frac{1}{2} \bar{\Theta}_{S} d V, & d \Theta_{C} & =d \theta+\frac{1}{2} d V \Theta_{S}, \\
d \bar{\Theta}_{S}=\frac{1}{2} \bar{\Theta}_{C} \tau_{3} d V, & d \Theta_{S}= & -\frac{1}{2} \tau_{3} d V \Theta_{C} .
\end{array}
$$


We express (A.24) as

$$
\begin{aligned}
& \delta \bar{\Theta}_{C}=\delta \bar{\theta}+d \bar{\Sigma}_{C}^{1}(\delta \theta)+\bar{\Sigma}_{C}^{2}(\delta \theta) \\
& \delta \bar{\Theta}_{S}=d \bar{\Sigma}_{S}^{1}(\delta \theta)+\bar{\Sigma}_{S}^{2}(\delta \theta)
\end{aligned}
$$

where $\bar{\Sigma}(\delta \theta)$ 's are arranged so that they do not contain $d(\delta \theta)$. The expressions for $\bar{\Sigma}(\delta \theta)$ 's are

$$
\begin{aligned}
& \bar{\Sigma}_{C}^{1}(\delta \theta)=\sum_{n=0} \frac{2 n+2}{(4 n+5) ! !}\left[\bar{\Theta}_{2 n+1} V_{\theta, \delta \theta}+(2 n+1) \bar{\Theta}_{2 n}\left(V_{\theta, \delta \theta} V\right)\right]\left(-\tau_{3}\right)^{n+1} \\
& \bar{\Sigma}_{S}^{1}(\delta \theta)=\sum_{n=0} \frac{2 n+1}{(4 n+3) ! !}\left[-\bar{\Theta}_{2 n} V_{\theta, \delta \theta}+2 n \bar{\Theta}_{2 n-1}\left(V_{\theta, \delta \theta} V\right)\right]\left(-\tau_{3}\right)^{n+1}
\end{aligned}
$$

and $\bar{\Sigma}^{2}$ s s are related to $\bar{\Sigma}^{1}$ s by

$$
\begin{aligned}
& \bar{\Sigma}_{C}^{2}(\delta \theta)=\frac{1}{2} \bar{\Sigma}_{S}^{1}(\delta \theta) d V-\bar{\Theta}_{S} V_{\delta \theta, d \theta}, \\
& \bar{\Sigma}_{S}^{2}(\delta \theta)=\left(-\frac{1}{2} \bar{\Sigma}_{C}^{1}(\delta \theta) d V+\bar{\Theta}_{C} V_{\delta \theta, d \theta}\right) \tau_{3} .
\end{aligned}
$$

Corresponding relations for even variation is from (A.22)

$$
\begin{aligned}
\Delta \bar{\Theta}_{j}= & -(-)^{j} \frac{2 j+1}{2} \bar{\Theta}_{j-1} \Delta V \\
& +(j+1)(j-1) \bar{\Theta}_{j-2}(V \Delta V) \\
& -(-)^{\frac{j(j-1)}{2}}\left\{\frac{1}{2} \bar{\theta} \Delta V-\Delta \bar{\theta} V\right\} V^{j-1}, \quad j \geq 1 .
\end{aligned}
$$

where

$$
\Delta V=V_{\Delta \theta, d \theta}+V_{\theta, \Delta d \theta}=-d V_{\theta, \Delta \theta}+2 V_{\Delta \theta, d \theta} .
$$

From (A.24)

$$
\begin{aligned}
\Delta \bar{\Theta}_{C} & =\Delta \bar{\theta}-\frac{1}{2} \bar{\Theta}_{S} \Delta V \\
& +\sum_{n=1} \frac{(2 n+1)(2 n-1)}{(4 n+1) ! !} \bar{\Theta}_{2 n-2}\left(-\tau_{3}\right)^{n}(V \Delta V) \\
& -\sum_{n=1} \frac{1}{(4 n+1) ! !}\left\{\frac{1}{2} \bar{\theta} \Delta V-\Delta \bar{\theta} Y\right\} V^{2 n-1} \tau_{3}{ }^{n} \\
\Delta \bar{\Theta}_{S} & =-\frac{1}{2} \bar{\Theta}_{C} \tau_{3} \Delta V \\
& +\sum_{n=1} \frac{(2 n+2) 2 n}{(4 n+3) ! !} \bar{\Theta}_{2 n-1}\left(-\tau_{3}\right)^{n+1}(V \Delta V) \\
& +\sum_{n=0} \frac{1}{(4 n+3) ! !}\left\{\frac{1}{2} \bar{\theta} \Delta V-\Delta \bar{\theta} V\right\} V^{2 n} \tau_{3}{ }^{n+1} .
\end{aligned}
$$


Corresponding to (A.28) we write them as

$$
\begin{aligned}
\Delta \bar{\Theta}_{C} & =\Delta \bar{\theta}+d \bar{\Sigma}_{C}^{1}[\Delta \theta]+\bar{\Sigma}_{C}^{2}[\Delta \theta], \\
\Delta \bar{\Theta}_{S} & =d \bar{\Sigma}_{S}^{1}[\Delta \theta]+\bar{\Sigma}_{S}^{2}[\Delta \theta],
\end{aligned}
$$

where $\Sigma^{1}[\Delta \theta]$ is obtained from $\Sigma^{1}(\delta \theta)$ by replacing $\delta \theta$ by $c \Delta \theta$ and remove $c$ from the left and put minus on it (due to the existence of $d$ on it in the definition).

$$
\begin{aligned}
& \bar{\Sigma}_{C}^{1}[\Delta \theta]=\sum_{n=0} \frac{2 n+2}{(4 n+5) ! !}\left[\bar{\Theta}_{2 n+1} V_{\theta, \Delta \theta}-(2 n+1) \bar{\Theta}_{2 n}\left(V_{\theta, \Delta \theta} V\right)\right]\left(-\tau_{3}\right)^{n+1} \\
& \Sigma_{S}^{1}[\Delta \theta]=\sum_{n=0} \frac{2 n+1}{(4 n+3) ! !}\left[\bar{\Theta}_{2 n} V_{\theta, \Delta \theta}+2 n \bar{\Theta}_{2 n-1}\left(V_{\theta, \Delta \theta} V\right)\right]\left(-\tau_{3}\right)^{n+1}
\end{aligned}
$$

$\bar{\Sigma}^{2}[\Delta \theta]$ is obtained from $\bar{\Sigma}^{2}(\delta \theta)$ and satisfies, from (A.31)

$$
\begin{aligned}
& \bar{\Sigma}_{C}^{2}[\Delta \theta]=-\frac{1}{2} \bar{\Sigma}_{S}^{1}[\Delta \theta] d V-\bar{\Theta}_{S} V_{\Delta \theta, d \theta}, \\
& \bar{\Sigma}_{S}^{2}[\Delta \theta]=\left(\frac{1}{2} \bar{\Sigma}_{C}^{1}[\Delta \theta] d V-\bar{\Theta}_{C} V_{\Delta \theta, d \theta}\right) \tau_{3} .
\end{aligned}
$$

The variations of $\Sigma^{1}[g]$ 's under even variation $\Delta_{f} \theta=f$ is expressed as

$$
\begin{gathered}
\Delta_{[f}\left(\bar{\Sigma}_{C}^{1}\left[g_{]}\right]\right) \equiv d \Lambda_{C}^{1}+\Lambda_{C}^{2}, \\
\Delta_{[f}\left(\bar{\Sigma}_{S}^{1}\left[g_{]}\right]\right) \equiv d \Lambda_{S}^{1}+\Lambda_{S}^{2}
\end{gathered}
$$

so that $\Lambda$ 's do not contain $d f$ and $d g$. The explicit form of $\Lambda$ 's are

$$
\begin{aligned}
\Lambda_{C}^{1}= & \sum_{n=0} \frac{(2 n+2)(2 n+1)}{(4 n+5) ! !}\left[\frac{1}{2} \bar{\Theta}_{2 n} V_{\theta,[f} V_{\theta, g]}-2 n \bar{\Theta}_{2 n-1} V_{\theta,[f}\left(V V_{\theta, g]}\right)\right. \\
& \left.+2 n(2 n-1) \bar{\Theta}_{2 n-2}\left(V V_{\theta, f}\right)\left(V V_{\theta, g}\right)\right]\left(-\tau_{3}\right)^{n+1} \\
\Lambda_{S}^{1}= & \sum_{n=1} \frac{(2 n+1)(2 n)}{(4 n+3) ! !}\left[\frac{1}{2} \bar{\Theta}_{2 n-1} V_{\theta,[f} V_{\theta, g]}+(2 n-1) \bar{\Theta}_{2 n-2} V_{\theta,[f}\left(V V_{\theta, g]}\right)\right. \\
& \left.+(2 n-1)(2 n-2) \bar{\Theta}_{2 n-3}\left(V V_{\theta, f}\right)\left(V V_{\theta, g}\right)\right]\left(-\tau_{3}\right)^{n+1} .
\end{aligned}
$$

$\Lambda^{2}$ 's are related to $\Lambda^{1}$ 's by

$$
\begin{aligned}
\Lambda_{S}^{2} & =-\frac{1}{2} \Lambda_{C}^{1} \tau_{3} d V-\bar{\Theta}_{C} \tau_{3} V_{f, g}-\bar{\Sigma}_{C}^{1}\left[[g] \tau_{3} V_{f], d \theta},\right. \\
\Lambda_{C}^{2} & =\frac{1}{2} \Lambda_{S}^{1} d V+\bar{\Theta}_{S} V_{f, g}-\bar{\Sigma}_{S}^{1}\left[[g] V_{f], d \theta} .\right.
\end{aligned}
$$


Finally we give lists of "signs" when spinors are interchanged in the bi-linear forms in table.6.16.

Table 2: Signs of bi-spinor forms under transposition

\begin{tabular}{|c|c|c|c|c|}
\hline \multirow[b]{2}{*}{ bi-linear forms } & \multicolumn{4}{|c|}{$(\operatorname{sign}) \quad$ for $(\chi, \phi)$ with their sign } \\
\hline & (odd,odd) & (odd,even) & (even,odd) & (even,even) \\
\hline $\bar{\chi} \mathcal{S}_{1} \phi=(\operatorname{sign}) \bar{\phi} \mathcal{S}_{1} \chi$ & - & - & - & + \\
\hline $\bar{\chi} \mathcal{C}_{1} \phi=(\operatorname{sign}) \bar{\phi} \mathcal{C}_{1} \chi$ & - & + & + & + \\
\hline \multicolumn{5}{|l|}{ for odd $V$} \\
\hline $\bar{\chi} \mathcal{S}_{1} \bigvee \phi=($ sign $) \bar{\phi} \bigvee \mathcal{S}_{1} \chi$ & - & + & + & + \\
\hline $\bar{\chi} \mathcal{C}_{1} \bigvee \phi=(\operatorname{sign}) \bar{\phi} \bigvee \mathcal{C}_{1} \chi$ & + & + & + & - \\
\hline \multicolumn{5}{|l|}{ for even $V$} \\
\hline $\bar{\chi} \mathcal{S}_{1} \bigvee \phi=($ sign $) \bar{\phi} \bigvee \mathcal{S}_{1} \chi$ & + & + & + & - \\
\hline $\bar{\chi} \mathcal{C}_{1} V \phi=(\operatorname{sign}) \bar{\phi} \bigvee \mathcal{C}_{1} \chi$ & + & - & - & - \\
\hline
\end{tabular}




\section{References}

[1] M.B.Green,J.H.Schwarz and E.Witten, "Superstring theory 1, 2", Cambridge University Press (1987).

[2] J.Polchinski, Phys. Rev. Lett. 75 (1995) 4724;

TASI Lectures on D-Branes, hep-th/9611050.

[3] E.Witten, Nucl.Phys. B460 (1995) 335.

[4] M.Aganagic, C. Popescu and J.H.Schwarz, Phys. Lett. 393B (1997) 311.

[5] M.Aganagic, C. Popescu and J.H.Schwarz, Nucl. Phys. B495 (1997) 99.

[6] M.Aganagic, C. Popescu and J.H.Schwarz, Nucl. Phys. B496 (1997) 215.

[7] M.Cederwall, A.von Gussich, B.E.W.Nilsson and A.Westerberg, Nucl. Phys. B490 (1997) 163;

M.Cederwall, A.von Gussich, B.E.W.Nilsson, P.Sundell and A.Westerberg, Nucl. Phys. B490 (1997) 179;

[8] E.Bergshoeff and P.K.Townsend, Nucl. Phys.490B (1997) 145.

[9] R.Kallosh, Phys. Rev. D56 (1997) 3515;

E.Bergshoeff, R.Kallosh, T.Ortin and G.Papadopoulos, Nucl. Phys. B502 (1997) 149 ;

R.Kallosh, "Volkov-Akulov theory and D-branes", hep-th/9705118;

R.Kallosh, "Quantization of p-branes, D-p-branes and N-branes", hep-th/9709202.

[10] M.Hatsuda and K.Kamimura, "Covariant Quantization Super D-string", hepth/9708001, to appear in NPB.

[11] J.A.de Azcárraga, J.M.Izquierdo and P.K.Townsend, Phys. Lett.B267 (1991) 366.

[12] J.Lee, "Hamiltonian formulation of D-brane action and the light-cone Hamiltonian", hep-th/9708093.

[13] J.A.de Azcárraga, J.P.Gauntlett, J.M.Izquierdo and P.K.Townsend, Phys. Rev. Lett. 63 (1989) 2443;

[14] T.Banks, N.Seiberg and S.Shenker, Nucl. Phys. B490 (1997) 91.

[15] C.M.Hull, "Gravitational Duality, Branes and Charges", Hep-th/970562 (1997).

[16] D.Sorokin and P.K.Townsend, "M-theory superalgebra from the M-5-brane", hepth/9708003 (1997).

[17] K.Kamimura, Nuovo Cimento 68B (1982) 33.

[18] S.Bergshoeff and E.Sezgin, Phys. Lett. B354 (1995) 256.

[19] H. Hammer, "Topological Extensions of Noether Charge Algebras carried by D-pBranes", hep-th/9711009.

[20] M.Hatsuda and K.Kamimura, "Wess-Zumino actions for IIA D-p-branes and their supersymmetries", in preparation. 